\title{
GRAIN SELECTION DURING SOLIDIFICATION IN SPIRAL GRAIN SELECTOR
}

\author{
H.J. Dai ${ }^{1}$, J-C. Gebelin², M. Newell ${ }^{2,3}$, R.C. Reed ${ }^{2}$, N.D’Souza ${ }^{3}$, P.D. Brown ${ }^{3}$, H.B. Dong ${ }^{1,3}$ \\ ${ }^{1}$ Department of Engineering, University of Leicester, University Road, Leicester LE1 7RH, UK \\ ${ }^{2}$ Department of Materials, University of Birmingham, Birmingham, B15 2TT, UK \\ ${ }^{3}$ Rolls-Royce plc, P.O. Box 31, Derby, DE24 8BJ, UK
}

Keywords: Grain selection, Directional solidification, Single crystal, Solidification modelling, CMSX-4

\begin{abstract}
A macro-scale ProCAST and a meso-scale Cellular Automaton Finite Element model (CAFE) are used to simulate the competitive growth and grain selection during solidification in spiral grain selector, which is widely used in industry to produce single crystal turbine blades. The macro-model ProCAST calculates the macroscopic heat transfer and fluid flow and the calculated thermal profiles are then used as input in the meso-scale CAFE model to predict grain structures and grain orientations. Competitive growth and grain selection in the selector has been analysed with emphasis on the shape of the spiral. It is found that the spiral becomes more effective as a grain selector, when it has a smaller "take-off" angle. However, the grain orientations cannot be optimised during the selection event in the spiral. To validate the modelling results, spirals with different shapes were cast in a fully instrumented industrial directional casting furnace and the grain structure and orientations in the spirals were analyzed.
\end{abstract}

\section{Introduction}

The operating temperature of turbine blades in jet engines has progressively increased because of the demand for ever higher turbine entry temperatures in the engine to save energy and increase thrust. Turbine blades are made from Ni-base superalloys. It is well known that theses alloys are difficult to forge, in particular when the blades have complex geometries with intricate channels which allow cooler air flow within and along the blades during operation [1-2]. Therefore, turbine components are usually produced by investment casting, particularly rotators such as blades and static components like nozzle guide vanes and seal segments. During casting two types of grain structures are formed in the blades: columnar grains and equiaxed grains [3-9] (see Fig 1). Fig 1(a) is a Ni-base superalloy turbine blade, which was produced by investment casting, i.e., by pouring the molten metal into a consolidated ceramic mould. After chemical etching, the grain structure of this blade is clearly revealed by zones of different grey levels. As can be seen, the crystals grow approximately equally in their preferred growth directions, forming roughly rounded-shaped, equiaxed grains. Fig 1(b) is a turbine blade with an array of parallel aligned dendrites from the bottom of the blade to the top. The parallel elongated dendrites constitute a region of columnar grains. To grow the columnar grains in the blades, a positive thermal gradient is imposed at the growth front. This is achieved through directional solidification where heat is extracted through the solid, in the opposite direction to the growth direction (see Fig 2 (a)). Directional solidification (DS) furnaces have been used to grow blades with columnar grains. As shown in Fig 2 (a), the DS furnace comprises 3 zones:
(1) a vacuum induction-melting unit; (2) a resistance-heated furnace chamber and (3) a withdrawal chamber. The preheated ceramic mould is open at the bottom and sits on a water-cooled copper chill plate. The molten superalloy solidifies on contact with the copper chill to form a thin layer of equiaxed grains. Subsequent growth favors those grains where the $<001>$ direction is most closely aligned with the thermal gradient, producing an array of columnar grains with a common vertical $<001>$ crystal orientation. After solidification starts, the water-cooled chill plate is lowered gradually. Withdrawal of the ceramic shell mould filled with molten alloy from the heated furnace produces a blade with columnar grains as shown in Fig 1 (b).

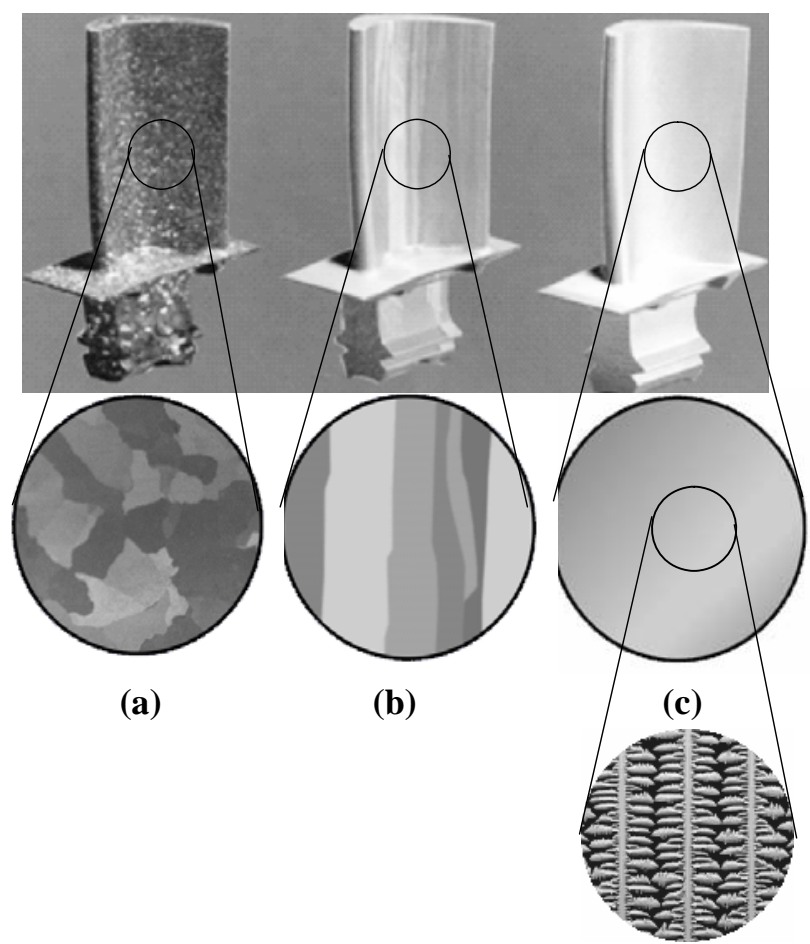

(d)

Fig 1 Ni-base superalloy turbine blades solidified as (a) equiaxed morphology, (b) columnar grains, (c) single crystal, (d) an enlarged view of the single crystal blade showing detailed dendritic structure calculated using CAFD solidification software [7].

To completely remove high angle grain boundaries and to gain exact control of grain orientation in columnar blades, blades with a 
single grain is desired. In practice, casting a single crystal blade is achieved by directional solidification combined with a spiral grain selector and a starter block as schematically shown in Fig 2.

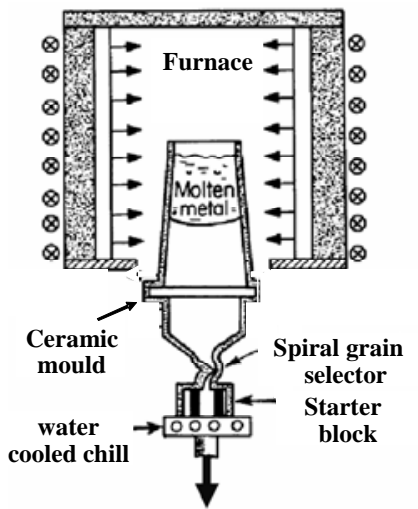

(a)

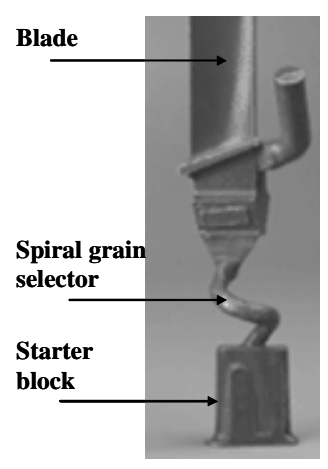

(b)
Fig. 2 (a) Schematic drawing of investment casting for single crystal turbine blade, (b) single crystal turbine blade with spiral grain selector [1].

In casting foundries, a prefabricated starter block is placed at the bottom of the mould (see Fig 2 (a)). The mould is withdrawn from the furnace to grow the blade. Several grains nucleated within the starter block can grow into the spiral-shaped passageway (called spiral grain selector) and most of them will be eliminated. Invariably only one grain survives during competitive growth in the spiral grain selector. If solidification in the main body initiates from a single crystal at the top of the spiral, then the whole blade solidifies as a single-crystal, which is further aided if a high thermal gradient and low velocity is maintained during the growth of the blade.

As illustrated in Fig. 2(b), a grain selector consists of two parts: a starter block unit referring to competitive growth for the grain orientation optimisation and a spiral grain selector facilitating dendrite branching to ensure that only single grain eventually survives at the top of the seed. The grain selection at the starter block has been systematically studied by Carter et al [10] to predict the grain size and grain texture. The purpose of this study is therefore to simulate and analyse the competitive growth and grain selection in the selector with emphasis on the shape of the spiral. The effect of spiral geometry on the grain selection was simulated using a coupled model and then validated against experimental observation.
Parameters used in the spiral design are illustrated in Fig. 3 including the angle of the spiral $(\theta)$, corresponding pitch length $\left(L_{P}\right)$, the diameter of the spiral $\left(d_{S}\right)$ and thickness of the spiral $\left(d_{T}\right)$.

Table I shows the geometries of the spirals designed in this study. This includes 16 cases which are divided into two groups. The diameter of the base $\left(d_{B}\right)$ is chosen as $20 \mathrm{~mm}$ according to the common industrial practice.

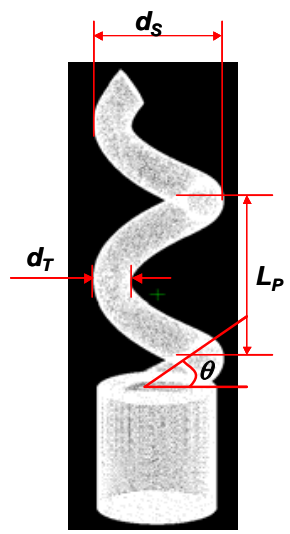

Fig. 3 Illustration of the grain selector, showing the parameters used in the spiral design.

\section{Simulation}

A macro-scale ProCAST model and a meso-scale Cellular Automaton Finite Element (CAFE) model are used to simulate the grain selection during directional solidification of single crystal investment castings, which are now widely used for a number of critical applications in gas turbine engines. The macro-model ProCAST is to simulate the macroscopic heat transfer and fluid flow by using a finite element solution of energy, momentum equations to determine the heat and momentum flow. Calculated thermal profiles are then coupled to the meso-model CAFE as input to predict grain structures and grain orientations in the grain selector.

\section{Orientation Selection in Starter block}

The thermal field plays a key role in casting. During directional solidification, the heat transfer in the grain selector was calculated using a VeriCast model [11] in ProCAST. The VeriCast model has been validated using a fully instrumented industrial directional casting furnace [11]. Experimentally measured temperature / time boundary conditions and the second-generation single crystal superalloy CMSX-4 with temperature dependent materials

Table I. Geometry of spirals designed in this study

\begin{tabular}{|c|c|c|c|c|c|c|c|c|c|c|c|c|c|c|c|c|}
\hline & \multicolumn{8}{|c|}{ Group A } & \multicolumn{8}{|c|}{ Group B } \\
\hline Case & 1 & 2 & 3 & 4 & 5 & 6 & 7 & 8 & 9 & 10 & 11 & 12 & 13 & 14 & 15 & 16 \\
\hline$\theta\left({ }^{\circ}\right)$ & 20 & 25 & 30 & 35 & 40 & 50 & 60 & 70 & 20 & 25 & 28 & 29 & 35 & 40 & 50 & 70 \\
\hline$L_{P}(\mathrm{~mm})$ & 6.5 & 8.5 & 10.5 & 12.8 & 15 & 21.5 & 31.5 & 50 & 7.5 & 9.3 & 10.5 & 11.2 & 14 & 17 & 24 & 55 \\
\hline$d_{s}(\mathrm{~mm})$ & \multicolumn{8}{|c|}{12} & \multicolumn{8}{|c|}{15} \\
\hline$d_{T}(\mathrm{~mm})$ & \multicolumn{8}{|c|}{3} & \multicolumn{8}{|c|}{5} \\
\hline
\end{tabular}


properties were used as inputs in this study. The initial temperature was set to $1475^{\circ} \mathrm{C}$. The shell was mounted on a watercooled copper chill base and surface nucleation density of $1 \times 10^{8}$ (number of nuclei $/ \mathrm{m}^{2}$ ) was applied to generate random nucleation at the bottom of the starter block. After soaking of the mould, the model assembly was withdrawn from the furnace at a predetermined rate and grains grew from the generated seeds at the bottom of the starter block. The withdrawal velocity during casting will affect the grain structure and defect formation. High withdrawal rates were found to generate a refinement of the microstructure and reduce the hot tearing tendency. More details about the effect of withdrawal velocity have been presented previously [12]. In this study, a withdrawal velocity of $0.06 \mathrm{mms}^{-1}$ is used during solidification. As an example, the calculated thermal profiles after $6 \mathrm{~s}$ solidification for the investment casting furnace assembly and the blade are shown in Figs. 4 (a) and (b). An enlarged view of the calculated thermal profile at the grain selector is shown in Fig 4 (c).

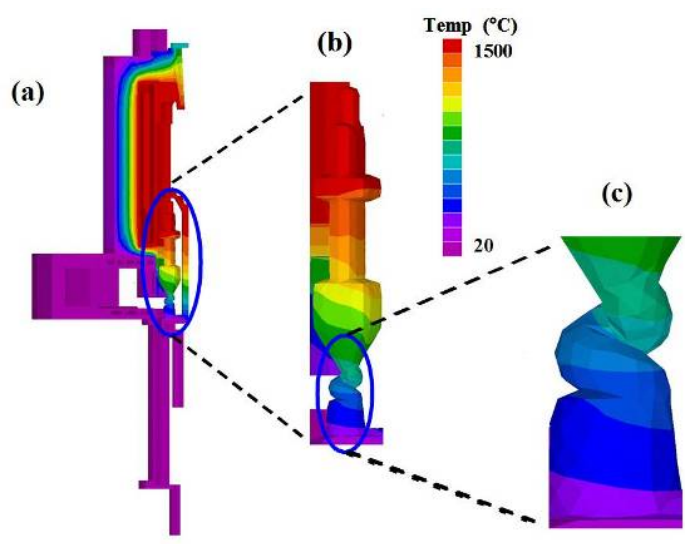

Fig. 4 Predicted three-dimensioned thermal fields during casting of turbine blades; (a) investment furnace, (b) enlarged view of the casting blade, (c) enlarged view of the grain selector.
The calculated thermal profiles are then used as inputs to predict grain structures using a grain structure model, cellular automaton finite element (CAFE) model [13-15]. CAFE couples a cellular automaton (CA) technique for grain growth with a finite element (FE) solver for heat flow. The advantages of the CAFE model are that the individual grains can be identified and the distribution in their shapes, sizes and orientations can be visualized.

Using the coupled model, predicted 3D grain structures and orientations in the starter block are shown in Fig. 5. The left picture (Fig. 5 (a)) is the predicted 3D grain structures in the grain selector including the starter block and the spiral. Different colours represent different grains characterized by different orientations. Figs. 5 (b1)-(e1) are the cross sections at different heights from the base along the solidification direction. At the bottom $(\mathrm{Y}=2 \mathrm{~mm})$, a large number of grains have nucleated with random orientations. During competitive growth accompanying solidification, the grains with orientations well aligned with the thermal gradient can grow faster than their unfavourably oriented neighbours and overgrow them and therefore lead to a decrease in the total grain number. At a height of $29 \mathrm{~mm}$ (Fig. 5 (e1)), a couple of grains were primarily selected after the competitive growth in the base and grew into the spiral, and then a single grain would eventually survive at a height corresponding to about one half of the pitch in the spiral.

The predicted $<001>$ pole figures showing the crystallographic texture in the starter block along the direction of heat flux at different heights from $2 \mathrm{~mm}$ to $29 \mathrm{~mm}$ are illustrated in the Figs. 5(b2)-(e2). At the bottom of the starter block, grains are random oriented and a $<001>$ texture develops within $10 \mathrm{~mm}$ from the bottom (Fig. 5(d2)), which agrees with the experimental observations and predictions by Carter et al [10].

Fig. 6 shows the average deviations of grain orientations at different heights from the bottom. This deviation is defined as the angle between the primary $<001>$ of the grain and the vertical (casting) axis. The dashed red line separates the starter block from the spiral grain selector. It is found that in the starter block the

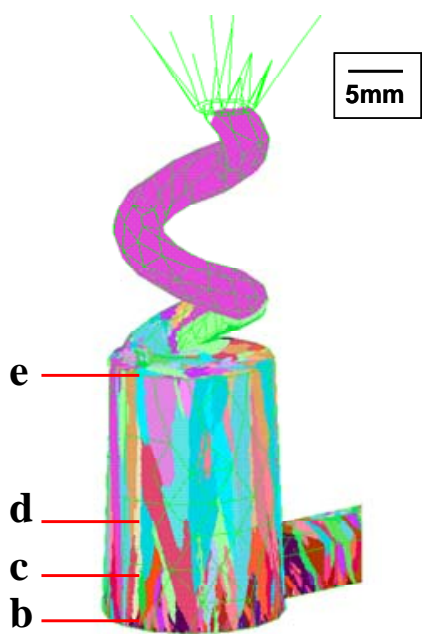

(a)

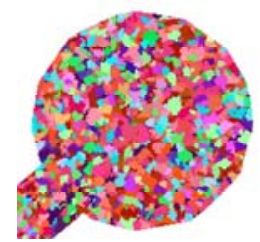

(b1)

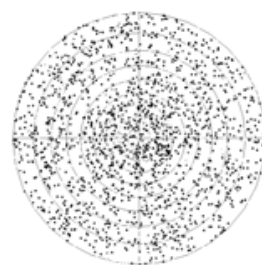

(b2)

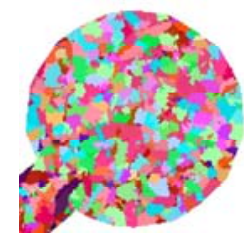

(c1)

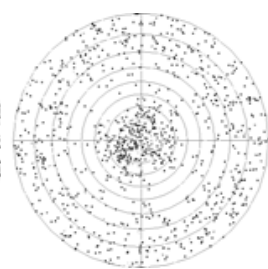

(c2)

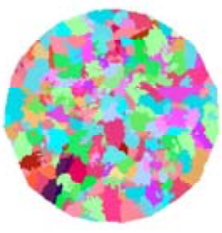

(d1)

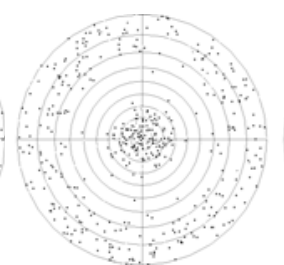

(d2)

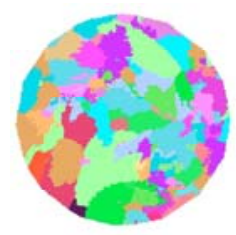

(e1)

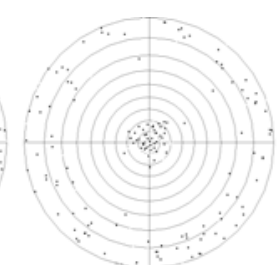

(e2)

Fig. 5 Predicted grain structure in the grain selector (a) and the cross sections at the height from the base of: (b1) 2mm; (c1) 5mm; (d1) 10mm; (e1) 29mm. Corresponding <001> pole figures at the height from the base of: (b2) 2mm; (c2) 5mm; (d2) 10mm; (e2) $29 \mathrm{~mm}$. 
average grain deviation decreases with the increase of the height along the solidification direction. The average deviation of grain orientations at the top of the cylindrical starter block can be controlled within $15^{\circ}$. Therefore, the starter block is effective in the selection of grain orientations.

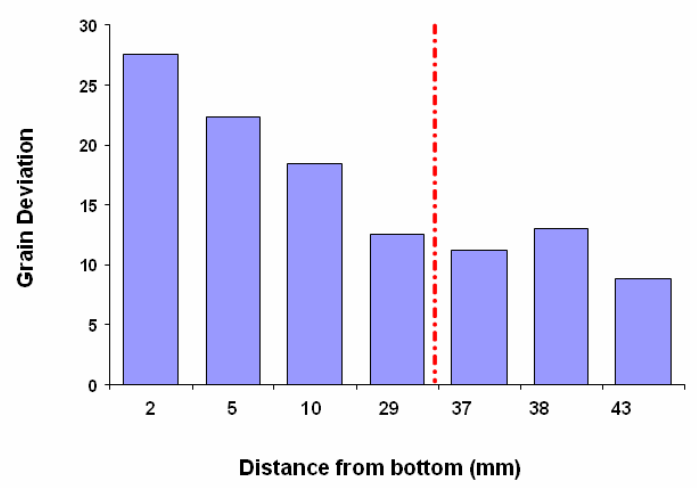

Fig. 6 Average deviations between $<001>$ direction of grains and the direction of the heat flux (casting axis) at different heights from the bottom.

We speculate that the function of the spiral is to select a single grain from those surviving grains at the top of the starter block. Consequently the spiral is used for grain selection, i.e. to ensure only one grain finally survives during growth within the spiral and subsequently grows into the turbine blade. To affirm our speculation, we designed spirals having different shapes to investigate the grain number and orientation selection within the spiral. For this purpose, the length of the starter block is reduced to $1 \mathrm{~mm}$ to enable more grains to be selected and to grow into the spiral.

\section{Grain Selection in Spiral Grain Selector}

Grain Number Selection in Spiral Grain Selector: It is of interest to investigate the efficiency of the spiral selector on the grain selection process with respect to different spiral designs. To achieve this, same initial solidification conditions were applied for all the cases shown in Table I.

Fig. 7 shows the simulated minimum heights where single grain texture is obtained for the spirals with different take-off angles, illustrating that grain selection depends on its geometry, especially the take-off angle $\theta$.

Single grain occurs at a higher location in the spiral with an increase of the take-off angle $\theta$. The reason for this can be explained as follows: With a smaller take-off angle, the crystals in the spiral have a less height to grow and have more chance to be blocked by the spiral wall. Smaller take-off angle corresponds to smaller pitch size, which means the cycle time for the spiral selection is also shorter and hence can improve the spiral efficiency. In addition, the smaller take-off angle $\theta$ offers more opportunity for dendrite branching, which also decreases the chance for grain survival and thereby makes grain selection more rapid than spirals designed with larger take-off angles. However, considering the diameter of the spiral itself, too small a take-off angle $\theta$ is not practical for spiral production. This probably explains why the take-off angle in the spiral selector is normally chosen between $25^{\circ}$ to $30^{\circ}$ in the single crystal casting process in industrial practice.

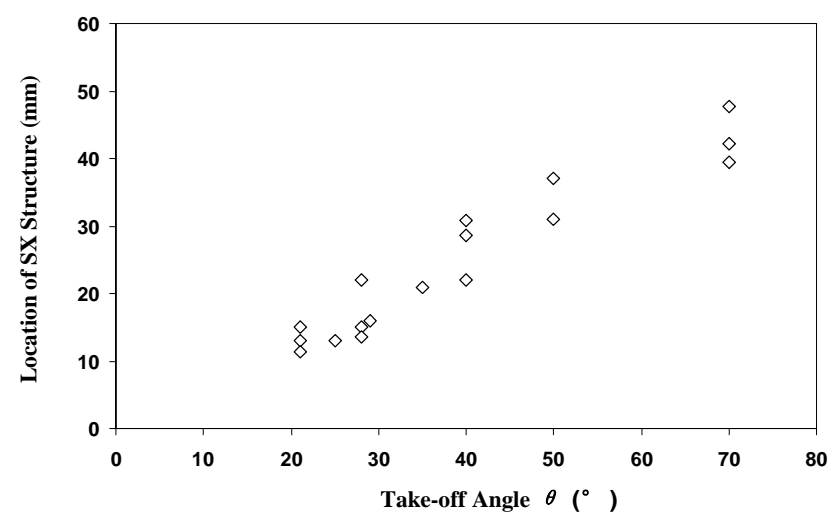

Fig. 7 Simulated minimum heights where single grain texture occurs in the spiral versus the take-off angle $\theta$.

The influences of the diameter of the spiral $\left(d_{S}\right)$ and thickness of the spiral $\left(d_{T}\right)$ on the efficiency of grain selection were also investigated and reported in ref [16], in excellent agreement with the results reported by $\mathrm{H}$. Esaka et al. [17].

Grain Orientation Selection in Spiral Grain Selector: The selection of grain numbers in the spiral selector has been discussed above and following is an investigation into the selection of grain orientations in the spiral.

As an example, Fig. 8 shows the simulated results for case 11 using the coupled ProCAST \& CAFE model. The initial surface nucleation at the bottom is $1 \times 10^{8}$ (number of nuclei $/ \mathrm{m}^{2}$ ), which yields $4 \times 10^{6}$ grains at the chill plate. During directional solidification, grain orientation selection occurred firstly in the $1 \mathrm{~mm}$ base and the selected grains then entered into the spiral. In the spiral, grain selection became much more efficient and one single grain was quickly selected below the position of one and a half pitches. As illustrated in Fig. 8, the average orientation deviations along the direction of $<001>$ of the grains which grew into the spiral is slightly optimized, since as discussed above the height of the base is critical for orientation selection. However, during the grain selection in the spiral, it seems that the spiral has no direct optimisation on grain orientations. To conclusively prove this, every single case in the spiral design was repeated 5 times for validation purposes. The results confirm that the orientation of the final SX grain is randomly selected in the spiral. More details can be explained in the pole figures below.

The pole figures in Fig. 8 clearly illustrate how the grain orientations are selected in the spiral during directional solidification. Fig. 8(a) shows the 3D simulated microstructure of the spiral for case 11, Figs. 8(b1) - (e1) are the standard <001> pole figures at different heights along the direction of heat flux, and Figs. 8(b2) - (e2) show the corresponding deviations of the grain orientations between the direction of the primary $<001>$ and the heat flux. 


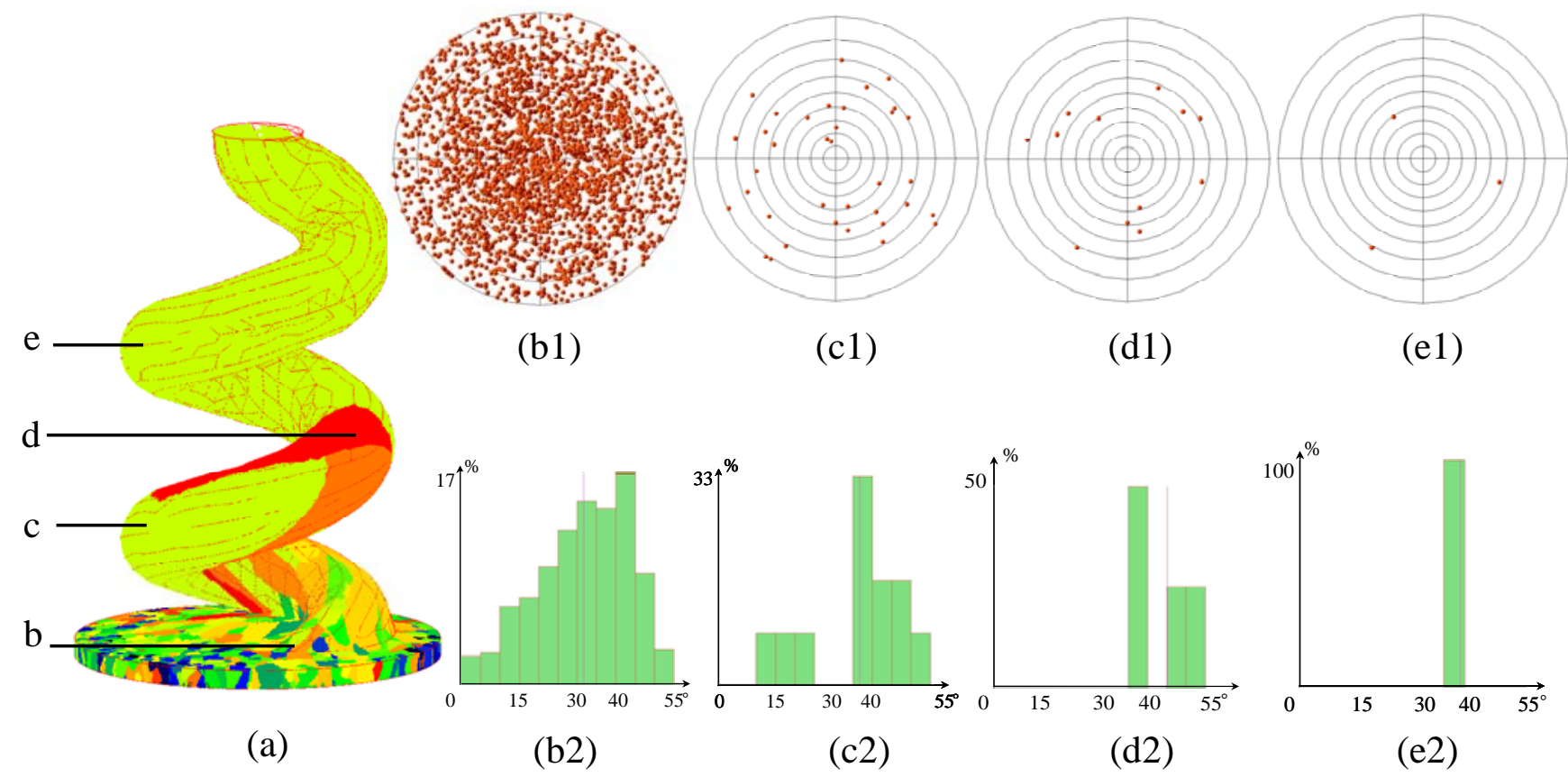

Fig. 8 Predicted grain structure in the spiral selector (a), $<001>$ pole figures along the direction of the heat flux at the height from the base of: (b1) $1 \mathrm{~mm}$; (c1) $6.25 \mathrm{~mm}$; (d1) 11.5mm; (e1) 16.75mm and the corresponding deviations between the direction of the grain primary $<001>$ and the heat flux ((b2)-(e2)).

Fig. 8(b1) shows the pole figure at the bottom of the spiral grain selector. It is found that the grain orientations are random and the $<001>$ texture is not obvious at this location. There would still be a large number of grains that would enter into the spiral. Fig. 8(b2) plots the distribution of the grain orientation deviations and the average grain orientation at the bottom of the spiral. Although the $1 \mathrm{~mm}$ base length is too short to produce the $<001>$ texture, the grain orientations have been slightly optimised and the average deviation of the grain orientations at the top of the base is $31^{\circ}$. This agrees with the previous discussion that the base is essential to select grains well aligned with the heat flux and the height of the base will determine the orientations of the grains which can enter the spiral. At the height of half pitch, the pole figure (Fig. 8 (c1)) shows that the number of grains has dramatically decreased. However, the points in the pole figure are still randomly distributed based on the orientations selected from the base. From the distribution of the grain deviations (Fig. 8 (c2)), the numbers of grains with orientations below $10^{\circ}$ and between $25^{\circ}-35^{\circ}$ have appreciably decreased rather than the grains with high deviations and the average deviation of the grain orientation became $37^{\circ}$, which was even higher than the previous stage. This proves that the overgrowth mechanism of grain selection in the spiral has no direct effect on the selection of the grain orientations. Therefore, the spiral does not play a role in the selection of the optimisation on grain orientations. Fig. 8(d1) shows the pole figure plotted at the height of one pitch. There are only four grains that survive after severe competition in the spiral. Compared with the grain orientation distribution at the height of half pitch, the grains with orientation deviations below $30^{\circ}$ are overgrown by the grains with high orientation deviations and no optimisation on the grain orientations is found (Fig. 8(d2)). At the height of one and a half pitches, the final SX grain was selected as shown in Fig. 8(e1). The orientation for the final SX grain is $39^{\circ}$, which overgrew the grains with either smaller or bigger deviations of grain orientations during solidification, and confirms the previous discussion about the selection of the grain orientations in the spiral. The texture evolution and the deviation of grain orientation analyses at different heights in the spiral confirm our proposal that the spiral grain selector is just used to rapidly select one grain and the grain orientation control occurs in the starter block.

\section{Experimental Investigation}

\section{Details of the Investment Casting Process Used}

Corresponding to different spiral grain selector designs, the casting experiments were conducted using a fully instrumented industrial directional casting furnace. Wax replicas were designed as shown in Fig. 9(a), consisting of spiral grain selectors and test bar sections as well as a few slim cylindrical bars to hold the spirals to maintain the original shapes, which would be finally removed before casting. Two or three spirals were assembled on one chill plate depending on the spiral geometries to economize the casting time. To achieve random nucleation, the spirals directly contact with the chill plate. Four castings were made with 8 different designed spiral selectors and 1 straight selector for comparison. Then the wax moulds were coated with multiple layers of ceramic slurry to obtain a ceramic shell. When the moulds were dry, the moulds were de-waxed in a steam autoclave, and then fired to remove all the residual wax and strengthen the ceramic shell by partial sintering. Before casting, CMSX-4 cylindrical charges were inserted into the test bar section on the top (Fig. 9(b)). The initial temperature for the casting was selected as $1570^{\circ} \mathrm{C}$ to ensure that the charges can melt completely. A series of water cooled baffles were used to keep heat flux perpendicular to the direction of withdraw. During solidification, 
a withdrawal velocity of $0.06 \mathrm{mms}^{-1}$ was maintained and the whole casting process took approximately four hours.
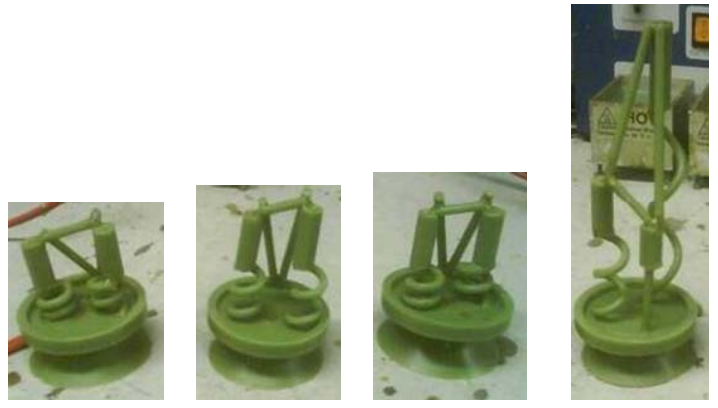

(a)
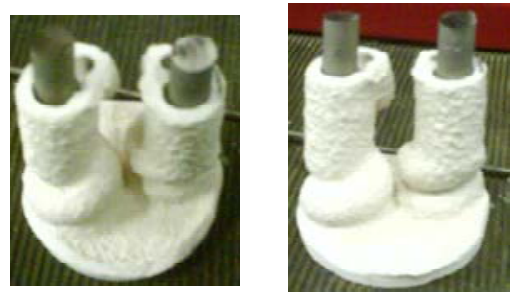

(b)

Fig. 9 Photographs of the wax assemblies prior to coating (a) and ceramic shells with charges before casting (b).

\section{Microstructure Analyses using Optical Microscopy}

To compare with the simulation results, different spirals were further analysed after casting. Using optical microscopy, the grain structures for different spirals produced during directional solidification were viewed and the efficiency of grain selection in the spiral corresponding to different spiral designs was investigated.

Fig. 10 shows the grain structures of the casting components for different spiral designs. The spirals were macro-etched using an electrolytic and chemical etch method routinely used in industrial practice before visual observation of the grain structures.

As illustrated in Fig. 10, at the bottom of the spiral, there are a large number of grains with random grain orientations and the grain size is very small. With competitive growth during solidification, the grain number quickly decreases and branching makes the grain size larger. Finally, a single grain survives. In Figs. 10(a) - (c), three different spiral castings are compared in terms of different take-off angles $\theta$, but having the same diameter $\left(d_{S}\right)$ and thickness $\left(d_{T}\right)$. A cylindrical bar with the same diameter as the spiral $\left(d_{S}\right)$ is also compared and shown in Fig. 10(d). As illustrated in Fig. 10, the location where single grain structure occurs differs. With the increase of the spiral pitch size, the single grain structure occurs at a later stage which means it will need more time to select the final grain and in turn decrease the efficiency of grain selection within the spiral. Compared with the spiral selector, the cylindrical bar selector does not successfully select one single grain after the similar length (shown in Fig. 10 (d)), which indicates that the spiral is more effective than the cylindrical bar in terms of selecting the grain numbers during directional solidification.

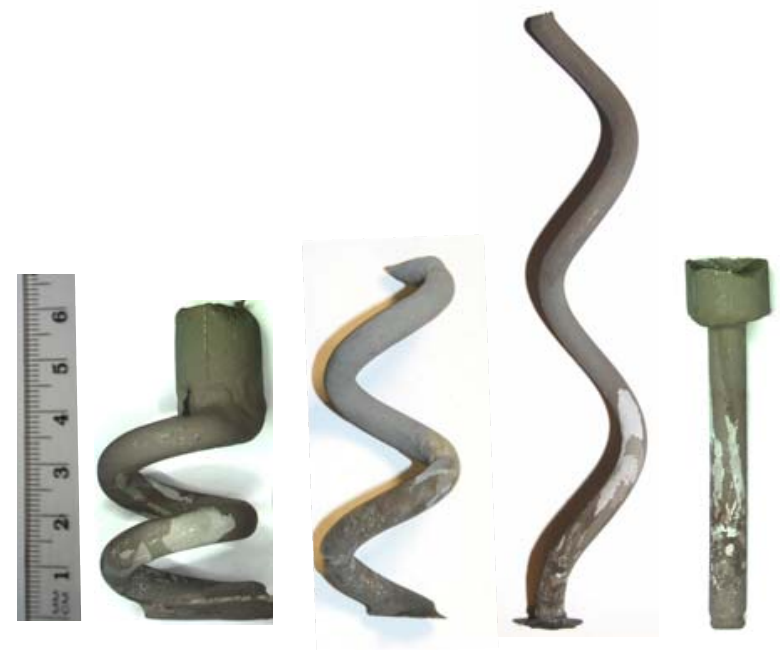

(a)

(b)

(c)

(d)

Fig. 10 The grain structures of the cast spirals in case: (a) 11; (b) 15; (c) 16 and a cylindrical bar for comparison.

The experimentally observed minimum heights where single crystal structure occurs are superimposed on the simulated results and shown in Fig 11. It reveals that the experimental observations agree well with our simulation results and confirms the importance of the spiral designs on the efficiency of grain selection during directional solidification.

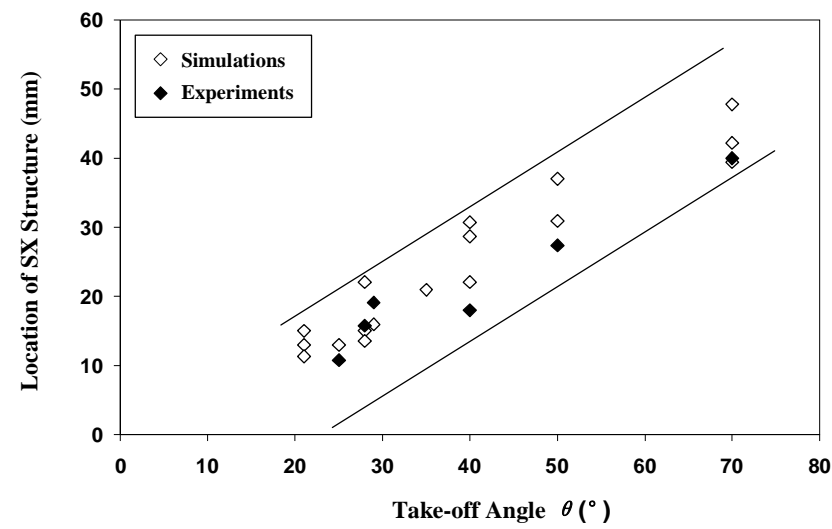

Fig. 11 Predicted and experimentally observed minimum heights where single grain structure occurs in spirals vs. take-off angle $\theta$

\section{Analyses of the Grain Orientations using EBSD}

To investigate the selection of grain orientations in the spiral and to compare with the simulation results, grain size and texture distributions at different heights of the spiral were analysed in this section.

Prior to analyses, the samples were cut at different heights vertical to the direction of heat flux. The surfaces of each section were polished to a $1 \mu \mathrm{m}$ finish and then polished for a further 10 minutes with colloidal silica. The prepared samples were then examined using a FEI Field Emission Gun Scanning Electron 


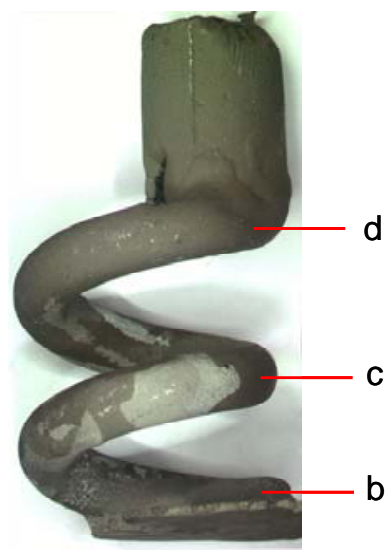

(a)

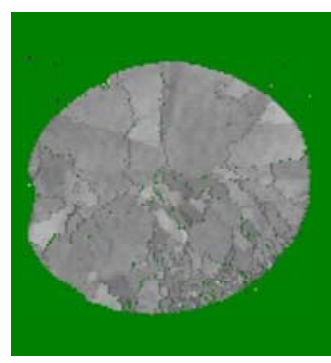

(b1)

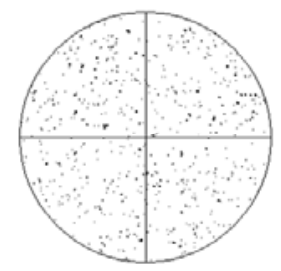

(b2)

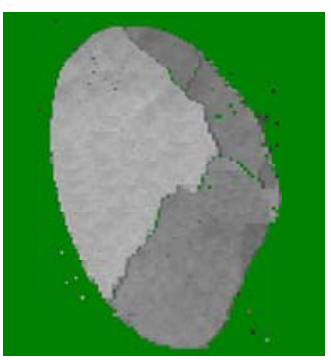

(c1)

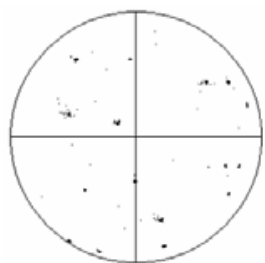

(c2)

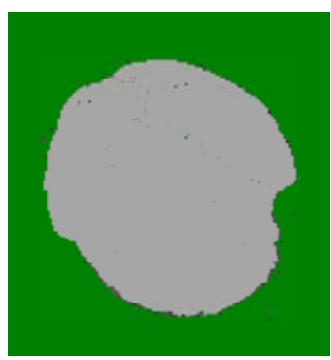

(d1)

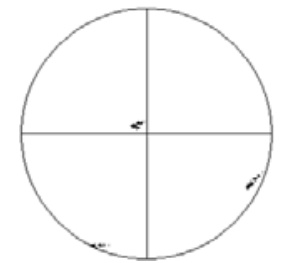

(d2)

Fig. 12 (a) optical photograph of the cast spiral, (b1)-(d1) the grain texture according to grain orientations at different heights of the spiral, (b2)-(d2) the corresponding <001> pole figures at the various heights as shown in (a).

Microscope (FEGSEM) by Oxford Instrument. Grain sizes and texture distributions were measured using Electron backscatter diffraction (EBSD). The instrument was made by HKL technology. The EBSD patterns were collected using a regular square grid of $50 \mu \mathrm{m}$, over the whole sample sections.

Fig. 12 shows the analysed results for the spiral in case 11 . Fig. 12(a) is an image taken using optical microscopy, Figs. 12(b1) (d1) show the grain texture of cross sections according to grain orientations at different heights of the spiral and Figs. 12(b2) - (d2) show the corresponding $<001>$ pole figures along the withdrawal direction. Fig. 12(b1) shows the grain texture at the section near the bottom of the spiral. The grey colour area stands for the sample. The grain boundaries can be seen clearly in the image and different grains were plotted using different gray colours. Sample b is cut at about $1 \mathrm{~mm}$ higher than the bottom of the spiral and shows a smaller number of grains compared with the simulation results. As shown in the pole figure along the withdrawal direction (Fig. 12(b2)), grain orientations are random with no preferred texture. Fig. 12(c1) shows the grain texture of the cross section at the height of one pitch. The grain numbers quickly decreased to 5 after one pitch length during competitive growth and the grain sizes were much coarser owing to grain growth and branching. The orientations of these 5 grains were plotted in the standard $<001>$ pole figures along the direction of heat flux shown in Fig. 12(c2). From the pole figure, we can find the grain orientations were randomly selected during the solidification and no preferred texture has been made during the grain selection. At the height of two pitches (Fig. 12(d1)), one single grain was finally selected and the pole figure was shown in Fig. 12(d2).

For comparison, Fig. 13 shows the simulation results of angles between the casting axis and the primary $<001>$ direction of the final single crystal corresponding to different take-off angles as shown in Table I. We observe that the grain orientations are randomly distributed and no optimisation occurs during grain orientation selection in the spiral. Both the simulation results and experimental analyses verify that the spiral can not optimise the grain orientation.

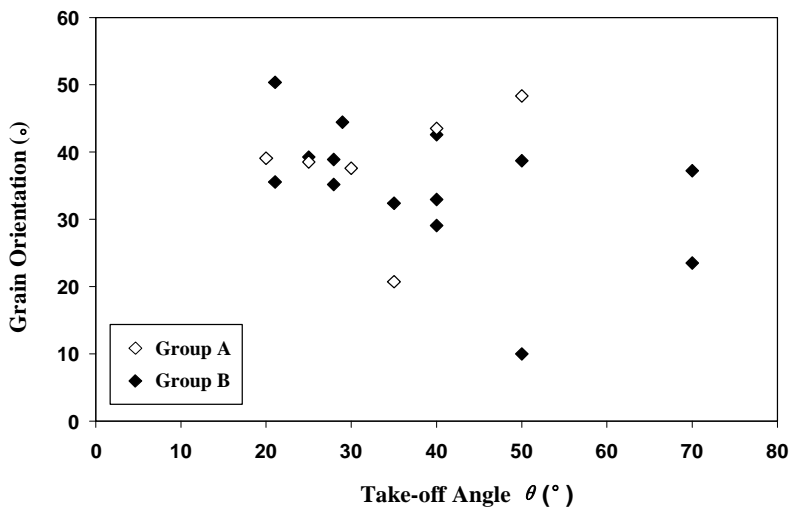

Fig. 13 Simulation results of angles between the direction of the heat flux during casting and $<001>$ direction of the final single crystal corresponding to different take-off angles

\section{Concluding Remarks}

Majority of the selection of grain orientation occurs in the starter block and only a couple of grains with well aligned orientations can grow into the spiral. The purpose of the spiral selector is only to rapidly but randomly select one single grain and the spiral efficiency significantly depends on the spiral take-off angle. 
Although the spiral is effective in selecting grain numbers, it has no direct effect on the grain orientation optimisation.

\section{Acknowledgements}

H. B. Dong acknowledges the Royal Society and EPSRC for offering him a Royal Society Industry Fellowship and RollsRoyce plc for hosting him at the Precision Casting Facility (PCF), Derby, UK.

\section{References}

1. R. C. Reed, The Superalloys Fundamental and Applications (Cambridge University Press, 2006), pp 18-25.

2. Yukiya Nakagawa, "Aero-Engine business and material technologies in Japan”, Superalloys 2004: 3-13.

3. W. Kurz and D. J. Fisher, Fundamentals of Solidification, Trans Tech Publications, Aedermannsdorf, Switzerland, 3rd Ed., 1989.

4. P. Caron and T. Khan, "Evolution of Ni-based superalloys for single crystal gas turbine blade applications. Aerospace Science and Technology", 1999. 3: p. 513-523.

5. John Campbell, Casting, Butterworth Heinemann, 2003

6. O.N. Dogan, "Columnar to equiaxed transition in high $\mathrm{Cr}$ white iron castings”, Scripta Materialia, 1996. 35(2): p. 163-168.

7. H.B. Dong and P.D. Lee, "Simulation of the columnar-toequiaxed transition in directionally solidified $\mathrm{Al}-\mathrm{Cu}$ alloys", Acta Mater., 2005. 53(3): p. 659-668.
8. W. Kurz and D.J. Fisher, "Dendrite growth at the limit of stability: tip radius and spacing”, Acta Mater., 29, (1981), 11 .

9. J.S. Langer, H. Muller-Krumbhaar, "Theory of dendritic growth-I”. Elements of a stability analysis, Acta Metall. 26 (1978) 1681.

10. P. Carter, D.C. Cox, C.A. Gandin, R.C. Reed, "Process modelling of grain selection during the solidification of single crystal superalloy castings”, Mater. Sci. Eng. A280 (2000) 233-246

11. M. J. Goulette, P. D. Spilling, R. P. Arthey, Cost effective single crystals, in: M.Gell, C.S. Kortovich, et al (Eds), Superalloys 1984, Metallurgical Society of AIME, Warrendale, PA, 1984, pp 167-176

12. Y.Z. Zhou, A. Volek and R. F. Singer, Influence of solidification conditions on castability of nickel-base superalloy IN792, Metall. Mater. Trans., 36A, (2005), pp 651-656

13. Ch.-A. Gandin and M. Rappaz, Acta Metall, 42, (1994), pp. 2233-2246

14. M. Rappaz and Ch.-A. Gandin, Acta Metall, 41, (1993), pp. 345-360

15. Ch.-A. Gandin, J.-L. Desbiolles, M. Rappaz and Ph. Thévoz, Metal Trans A, 30A, 1999, pp. 3153-3165

16. H.J. Dai, J-C. Gebelin, N.D'Souza, P.D. Brown and H.B. Dong, International Journal of Cast Metals Research, In press

17. H. Esaka, K. Shinzuka M. Tamura, Mater. Sci. Eng. A413414 (2005) 151-155 